\title{
Loss of LARP4B, an early event in the tumorigenesis of brain cancer?
}

\author{
Sarah Blagden ${ }^{1}$, Cornelius Schneider ${ }^{2}$, Utz Fischer ${ }^{2}$ \\ ${ }^{1}$ Department of Oncology, University of Oxford, Oxford, UK; ${ }^{2}$ Department of Biochemistry, University of Wuerzburg, Wuerzburg, Germany \\ Correspondence to: Sarah Blagden. Department of Oncology, University of Oxford, Old Road Campus Research Building, Roosevelt Drive, Oxford, \\ OX3 7DQ, UK. Email: sarah.blagden@oncology.ox.ac.uk.
}

Submitted Oct 18, 2016. Accepted for publication Oct 25, 2016.

doi: $10.21037 /$ tcr.2016.11.33

View this article at: http://dx.doi.org/10.21037/tcr.2016.11.33

In April 2016, Koso and colleagues from professor Sumiko Watanabe's group at University of Tokyo published a paper in Cancer Research describing LARPAB as a tumour suppressor gene (TSG) in glioma (1). Glioblastoma (GB) is the most common, aggressive and locally invasive form of glioma with a median survival from diagnosis of 12-15 months despite maximal treatment (2). Survival has improved little over the last 30 years and effective therapies are still a major unmet clinical need. Prof. Watanabe's group developed a two-step method based around Sleeping Beauty transposon mutagenesis to identify genes required for cancer initiation and progression. The group has previously used this technique to characterise bowel cancer development (3), but here they focused on gliomas. They first mutagenised neural stem cells (NSCs) in vitro into glioma-initiating cells that are believed to be the progenitors of gliomas. These cells were subcutaneously injected into the flanks of immunocompromised (SCID) mice to allow tumour development following a second round of mutagenesis. Using this technique, $L A R P 4 B$ was identified as one of over 80 likely TSGs capable of transforming NSCs (4). By interrogating The Cancer Genome Atlas (TCGA) database, the authors observed heterozygous LARP4B deletion in $80 \%$ primary GB samples in association with methylation-induced transcriptional silencing of the second $L A R P 4 B$ allele and observed a correlation between low $L A R P 4 B$ copy number and adverse patient survival outcome.

This is the first time the RNA binding protein (RBP) LARP4B has been associated with glioma. LARP4B was initially described in 2002 as a component of an mRNA translation-regulating complex in rat neuronal synapses
$(5,6)$. LARP4B belongs to a seven-member family of 33 La-related proteins (LARPs) and its closest paralogue 34 is LARP4 (also known as LARP4a) (7). The individual 35 functions of members of this evolutionarily conserved family 36 of proteins are gradually being elucidated. LARP1, 1B, 4, 4B and 6 are cytosolic factors involved in mRNA metabolism whilst LARP3 and 7 primarily function in transcription regulation. LARP4 and 4B have been shown to bind and stabilise target mRNAs via an N-terminal "La module", an RNA-binding motif comprised of a La domain and an adjacent RRM conserved across every member of the LARP family. Both LARP4 and 4B have also been separately identified as existing in complexes with poly(A)-binding protein (PABP) and the ribosome-associated factor RACK1. Whilst LARP1, LARP3 (also known as "genuine La") and LARP6 appear to be proto-oncogenic, LARP4 and 4B have tumour suppressive activity. In prostate and more recently breast cancer cell lines, depletion of LARP4 has been shown to induce cell migration, invasion and increase cell motility $(8,9)$. Here, Koso et al. demonstrate that over-expression of $L A R P 4 B$ suppresses tumour proliferation and induces mitotic arrest and apoptosis in glioma cell lines. After $L A R P 4 B$ over-expression, mRNA levels of the mitotic regulatory gene $C D K N 1 A$ and the Bcl2 family member $B A X$ are upregulated. Interestingly, these factors have also been described as LARP4B targets in HEK293 cells (10). This indicates that LARP4B controls their stability and/or translation.

The LARP4B phenotype is reminiscent of that of its cousin, LARP1. LARP1 is a proto-oncogene that protects cancer cells from apoptotic cell death by directly binding multiple mRNAs including BIK and BCL2 $(11,12)$. 34 35 
Whilst LARP1 destabilises the pro-apoptotic regulator $B I K$, it stabilises the anti-apoptotic BCL2 with the net result of avoiding apoptosis in cancer cells in which LARP1 is strongly upregulated. As a tumour suppressor, LARP4B exerts the opposite effect by facilitating cell death through apoptosis. It is interesting to observe that a number of pro- and anti-apoptotic BCL2-related factors are present in the LARP4B interactome as defined by Photoactivatable Ribonucleoside-Enhanced Crosslinking and Immunoprecipitation (PAR-CLIP) (10). This suggests that, like LARP1, LARP4B might exert a dual effect on its target mRNAs, by stabilising the proapoptotic but destabilising the anti-apoptotic transcripts. The BCL2 family of proteins that includes Bcl-xL (also known as BCL2L1), Bad, Bid, BIK and BAX are components of the intrinsic or mitochondrial pathway of cell death. On death signalling they translocate from the cytoplasm to the mitochondria to release cytochrome $\mathrm{C}$ and induce caspasemediated apoptosis. It is interesting to note the presence of numerous mitochondrially-associated genes in both the LARP1 and LARP4B interactomes (10,11), particularly in light of the recent finding that Drosophila LARP1 acts to track mRNAs to the mitochondrial outer membrane whereupon they are released for localised protein synthesis (13). It is possible this mRNA-tomitochondrial tracking function is conserved across all other translation-associated LARPs (including LARP1B, LARP4, LARP4B and LARP6), although this cannot be assumed with any certainty until their full interactomes are known.

The authors suggest that dysregulation of $L A R P 4 B$ expression is an early event in tumorigenesis. It is likely that all tumors undergo a pre-invasive period before becoming metastatic and invasive and knowledge of the cumulative events that drive malignant change are instrumental for screening and treatment. Stepwise tumorigenesis has been best characterised in colorectal cancer where morphological and genetic alterations occur sequentially (to genes including APC, DCC and finally TP53) as the preinvasive colonic polyp transitions to an invasive cancer. The location of gliomas within the cranial cavity and their tendency to be diagnosed at symptomatic presentation makes biopsy and pre-invasive characterization almost impossible in vivo; any understanding of the molecular events that precede GB are mainly derived from post-mortem studies. The strength of the mutagenesis system described here is its ability to recapitulate the stepwise genetic changes that precede cancer development. But it raises the question of whether it gives an accurate representation of events in vivo. It is known that 113 extrinsic pressures such as the stromal and immunological milieu significantly contribute to tumorigenesis, in addition to competitive pressures within a heterogeneous population of cells. Also there is still debate as to the cell of origin for glioblastoma, it is probable that this differs between subtypes and that early genetic events are unlikely to be shared between them.

The gene encoding $L A R P 4 B$ is carried on the short arm of chromosome 10 at position $10 \mathrm{p} 15.3$. Loss of heterozygosity (LOH) of chromosome 10 is commonly observed in primary and secondary gliomas at frequencies of $60-80 \%$. This is caused by complete loss of the chromosome or deletion of three "hot spots", one of which is 10p14-15, indicating that TSGs are carried in these loci. So far, those identified in 10p have included KLF6 (found on 10p15.2) and PFKFB3 (found on 10p15.1). Although deletion of chromosome 10 is more commonly observed in primary than secondary GBs, there is debate as to whether it is an early or late event. It is interesting to note that another gene identified from Koso's mutagenesis screen was MLLT10 also encoded on 10p (at 10p12.3) and previously associated (by SNP genotyping) with risk of meningioma (14). The data from other glioma studies support the authors' findings that a TSG located at $10 \mathrm{p}$ (like $L A R P 4 B$ ) is likely to be influential on tumour pathophysiology.

As information from TCGA and other sources has become available, gliomas have been subclassified into classical, proneural, neural and mesenchymal types. By correlating their genomic and morphological features, the authors aligned their mutagenized NSC cells with the "mesenchymal" subtype of GB. This subtype is characterised by frequent mutations to NF1, PTEN and TP53 as well as upregulation of genes associated with glycolysis and gluconeogenesis (e.g., ALDH1A) (15). Glycolysis and gluconeogenesis are both metabolic processes and, like the apoptotic regulators, genes encoding ALDH1A and other glucose-metabolism proteins are strongly enriched within the LARP4B interactome. Again this would position LARP4B within this cancer subtype and perhaps indicate that loss of $L A R P 4 B$ could activate tumorigenesis by de-repressing the expression of these genes.

While the authors find $L A R P 4 B$ deletion in $80 \%$ of GB cases, it is surprising this aberration has not previously been described in large genome-wide mapping studies in glioma (16). For many years, genes encoding RBPs were thought to have insignificant roles in pathogenesis 
and were largely over-looked. Work by Vogel (17) indicated a disparity between levels of gene and protein expression in GB cell lines suggesting a significant post-transcriptional contribution to gene expression. This contradiction to the central dogma is becoming accepted in cancer where dysregulation of RBPs, splicing factors, long non-coding (lnc) and micro (mi) RNAs are increasingly recognised as altering if not dominating gene expression (18). It is therefore possible that RBPs identified in previous tumor mapping studies were disregarded. Alternatively, the expression of many RBPs is post-transcriptionally regulated (often they are selfregulated) and they are infrequently mutated and therefore genetic analyses and/or mutational screens would fail to identify them. In that respect $L A R P 4 B$ is unusual as deletion/mutation of LARPs has not yet been convincingly shown in other diseases.

That a methylation "hit" to the second allele of LARP4B is required to inactivate the gene indicates a potential therapeutic avenue to reactivate $L A R P 4 B$ using methylation inhibitors. Epigenetic changes are frequently observed in tumor initiating cells and these cells can be reprogrammed by removal of the cancer specific methylation marks (19). In the context of an established tumor, it is unclear whether restoring one allele of $L A R P 4 B$ is likely to have any clinical impact. Without knowing the chronology of genetic mutations it is difficult to know whether extrinsic $L A R P 4 B$ is likely to reverse changes or whether its functional relevance has diminished as genetic alterations accumulate in the in vivo setting.

The LARP family are emerging as having fundamental roles in tumorigenesis and as potential drug targets. RBPs like the LARPs are powerful targets as they regulate the expression of entire networks of proteins and can significantly alter the behaviour of a cancer cell. It is possible that, with advances in whole proteome sequencing, where proteomics and genomics can be conducted in parallel, a more comprehensive picture of cancer biology will emerge. With this, the role of genes such as $L A R P 4 B$ will become more evident. Thus the development of drugs that target post-transcriptional regulators like LARP4B could broaden the therapeutic options for many cancers that have failed to benefit from the genome-centric approach that has hitherto dominated drug discovery. Here, Koso and team present intriguing evidence that loss of LARP4B tumor suppressor function is an important early event that occurs during glioma tumorigenesis.
None.

Footnote

Provenance: This is a Guest Editorial commissioned by the Section Editor Ning Huang (Department of Neurosurgery, the Second Affiliated Hospital of Chongqing Medical University, Chongqing, China).

Conflicts of Interest: The authors have no conflicts of interest to declare.

Comment on: Koso H, Yi H, Sheridan $\mathrm{P}$, et al. Identification of RNA-Binding Protein LARP4B as a Tumor Suppressor in Glioma. Cancer Res 2016;76:2254-64.

\section{References}

1. Koso H, Yi H, Sheridan P, et al. Identification of RNABinding Protein LARP4B as a Tumor Suppressor in Glioma. Cancer Res 2016;76:2254-64.

2. Kleihues P, Barnholtz-Sloan JS, Ohgaki H. Tumours of the nervous system. In: Stewart BW, Wild CP. editors. World Cancer Report 2014. Lyon: International Agency for Research on Cancer, 2014:511-21.

3. Takeda H, Wei Z, Koso H, et al. Transposon mutagenesis identifies genes and evolutionary forces driving gastrointestinal tract tumor progression. Nat Genet 2015;47:142-50.

4. Koso H, Takeda H, Yew CC, et al. Transposon mutagenesis identifies genes that transform neural stem cells into glioma-initiating cells. Proc Natl Acad Sci U S A 2012;109:E2998-3007.

5. Angenstein F, Evans AM, Settlage RE, et al. A receptor for activated $\mathrm{C}$ kinase is part of messenger ribonucleoprotein complexes associated with polyA-mRNAs in neurons. J Neurosci 2002;22:8827-37.

6. Schäffler K, Schulz K, Hirmer A, et al. A stimulatory role for the La-related protein $4 \mathrm{~B}$ in translation. RNA 2010;16:1488-99.

7. Stavraka C, Blagden S. The La-Related Proteins, a Family with Connections to Cancer. Biomolecules 2015;5:2701-22.

8. Bai SW, Herrera-Abreu MT, Rohn JL, et al. Identification and characterization of a set of conserved and new regulators of cytoskeletal organization, cell morphology and migration. BMC Biol 2011;9:54. 
9. Seetharaman S, Flemyng E, Shen J, et al. The RNAbinding protein LARP4 regulates cancer cell migration and invasion. Cytoskeleton (Hoboken) 2016. [Epub ahead of print].

10. Küspert M, Murakawa Y, Schäffler K, et al. LARP4B is an AU-rich sequence associated factor that promotes mRNA accumulation and translation. RNA 2015;21:1294-305.

11. Mura M, Hopkins TG, Michael T, et al. LARP1 posttranscriptionally regulates mTOR and contributes to cancer progression. Oncogene 2015;34:5025-36.

12. Hopkins TG, Mura M, Al-Ashtal HA, et al. The RNAbinding protein LARP1 is a post-transcriptional regulator of survival and tumorigenesis in ovarian cancer. Nucleic Acids Res 2016;44:1227-46.

13. Zhang Y, Chen Y, Gucek M, et al. The mitochondrial outer membrane protein MDI promotes local protein synthesis and $\mathrm{mtDNA}$ replication. EMBO J 2016;35:1045-57.

14. Egan KM, Baskin R, Nabors LB, et al. Brain tumor risk according to germ-line variation in the MLLT10 locus.

Cite this article as: Blagden S, Schneider C, Fischer U. Loss of $L A R P 4 B$, an early event in the tumorigenesis of brain cancer? Transl Cancer Res 2016. doi: 10.21037/tcr.2016.11.33
Eur J Hum Genet 2015;23:132-4. 274

15. Mao P, Joshi K, Li J, et al. Mesenchymal glioma stem 275 cells are maintained by activated glycolytic metabolism $\quad 276$ involving aldehyde dehydrogenase 1A3. Proc Natl Acad 277 Sci U S A 2013;110:8644-9. 278

16. Hoelzinger DB, Mariani L, Weis J, et al. Gene expression 279 profile of glioblastoma multiforme invasive phenotype $\quad 280$ points to new therapeutic targets. Neoplasia 2005;7:7-16. 281

17. Vogel C, Abreu Rde S, Ko D, et al. Sequence signatures 282 and mRNA concentration can explain two-thirds of 283 protein abundance variation in a human cell line. Mol Syst 284 Biol 2010;6:400. 285

18. Schwanhäusser B, Busse D, Li N, et al. Corrigendum: 286 Global quantification of mammalian gene expression $\quad 287$ control. Nature 2013;495:126-7. 288

19. Stricker SH, Feber A, Engström PG, et al. Widespread 289 resetting of DNA methylation in glioblastoma-initiating $\quad 290$ cells suppresses malignant cellular behavior in a lineagedependent manner. Genes Dev 2013;27:654-69. 